\title{
Submental intubation in oral and maxillofacial surgery - a prospective clinical study. An anesthetic challenge
}

\begin{abstract}
Submental intubation is an interesting alternative when short-term postoperative control of airway is desirable with the presence of undisturbed access to oral as well as nasal airways and good dental occlusion in maxillofacial trauma and also in assessment of nasolabial complex during elective orthognathic surgeries. Submental intubation with paramedian approach has been used in 60 cases from September 2009 to December 2015 in the Department of oral and maxillofacial surgery. Of 60 patients, 30 patients who had midfacial fractures at the Lefort II, a displaced nasal bone fracture and 30 patients who underwent elective orthognathic surgery were selected. After standard orotracheal intubation, paramedial skin incision was placed in the submental region and passage was created by blunt dissection from the submental region in to the floor of the mouth. The proximal end of the orotracheal tube was pulled through the floor of the mouth with a haemostatic clamp in to the submental area and stabilized with 1-0 silk sutures. At the end of the surgery, the tube was pulled back to the usual oral route. There were no peri-operative complications related to the procedure. Average duration of the procedure was around 7 minutes. Submental intubation is a simple technique associated with low rates of morbidity. It is an attractive alternative for maintenance of airway not only in the surgical management of maxillofacial trauma but also in elective orthognathic surgeries.
\end{abstract}

Keywords: intubation, submental route, midface fractures, orthognathic surgery, nasal surgery
Volume 5 Issue 2 - 2016

\author{
Venkata Ramana Murthy V,' Dr. \\ Chandrasekhar Krishna murthi ${ }^{2}$ \\ Senior lecturer, Department of Oral and Maxillofacial Surgery, \\ Anil Neerukonda Institute of Dental Sciences, India \\ ${ }^{2}$ Associate Professor, Department of Anesthesiology, NRI \\ Institute of Medical Sciences, Sangivalasa,Visakhapatnam-53| I62, \\ Andhra Pradesh, India
}

Correspondence: Venkata Ramana Murthy V, Consultant Ora and Maxillofacial Surgeon, Senior lecturer, Anil Neerukonda Institute of Dental Sciences, D.No. 39-22-37/I, Kalinga Nagar, Sangivalsa,Visakhapatnam, Andhra Pradesh, 530162, India, Email murthymaxfac@gmail.com

Received: June 03, 2016 | Published: July 19,2016

\section{Introduction}

Medicine is a fast developing science and progress has helped improve patient comfort and safety. Surgeries involving the maxillofacial region are traditionally done using nasal or oral endotracheal intubation. Surgical repair of the maxillofacial region requires modification of the standard anesthetic technique, as these procedures present a unique set of problems both for the anesthetist and for the surgeon.

Naso-endotracheal intubation is the most commonly preferred choice in the management of various cases of maxillofacial trauma and elective cosmetic procedures for Dentofacial deformities. However it is frequently contraindicated because of concurrent skull base fractures with associated risk of passage of tube in to the cranial fossa. In addition, the presence of naso-tracheal tube can interfere with the surgical reconstruction of fractures of the naso-orbito-ethmoidal complex. ${ }^{2}$

The surgeon needs an unobstructed field, and in most instances maxillo-mandibular fixation is required intra-operatively for adequate reduction of facial fractures. Achieving optimal occlusion is one of the fundamental aims of the oral and maxillofacial procedures and hence such patients cannot be managed with oro-endotracheal intubation. ${ }^{3,4}$ Considering all the factors, the next choice would be tracheostomy, which is considered by many to be the preferred route for airway management in patients with complex maxillofacial fractures. However, tracheostomy is associated with a significant number of complications like hemorrhage, subcutaneous emphysema, pneumothorax, tracheal stenosis, respiratory tract infections, decanulation and cosmetic disfigurement with significant morbidity. ${ }^{5,6}$
An alternative technique of airway management in craniomaxillofacial trauma, the SUBMENTAL route has been described in 1986 by ALTEMIR. ${ }^{7}$ Over the years the SUBMENTAL route for endotracheal intubation has undergone various modifications and is safely indicated in patients with midface and panfacial trauma with possible skull base fractures. ${ }^{8}$ With conventional nasal intubation, precise intraoperative assessment of the changes to the nasolabial complex, assessment of midlines, cant, and incisor display cannot be made accurately during concomitant orthognathic procedures, elective Lefort osteotomies, and Rhinoplasty procedures. ${ }^{9}$ Because of perceived advantages of submental intubation over conventional nasotracheal intubation we have used it in our unit as an alternative in both maxillofacial trauma and elective orthognathic procedures.

\section{Materials and methods}

This prospective clinical study was conducted from September 2009 to December 2015 after obtaining the clearance from the institutional ethical committee. Of 60 patients, 30 male patients who had midfacial fractures at the Lefort II, a displaced nasal bone fracture and 30 female patients who underwent elective orthognathic surgery were selected for the study.

\section{A. Inclusion criteria}

a. All patients between 18-50 yrs of age.

b. Patients with midfacial and naso-ethmoidal fractures.

c. Patients undergoing' elective orthognathic surgery.

\section{B. Exclusion criteria}

a. Patients with systemic illness. 
b. Those requiring long-term airway support.

c. Patients with multi-organ trauma.

d. Those with a known history of keloid formation.

The technique used for submental intubation was an adaptation of the general principles published by Hernandez Altemir. ${ }^{7}$

\section{Operative procedure for paramedian submental intubation}

After normal oral intubation with a cuffed flexometallic tube, an incision measuring $2 \mathrm{cms}$ approximately was made in the submental and paramedial region, parallel to the lower border of the mandible, and a finger's breadth from the lower border of the mandible (Figure 1). A curved hemostat was then passed from the submental incision through the subcutaneous layer, platysma, mylohyoid muscle, submucosal layer and mucosa (Figure 2). It enters the oral cavity at the junction of the attached lingual alveolar mucosa and the free mucosa of the floor of the mouth. An intraoral incision $1 \mathrm{~cm}$ in length was made parallel to the gingival soft tissue to create a tunnel for the introduction of the endotracheal tube.

A large curved hemostat was then placed through the submental incision. The pilot tube connector was grasped and pulled through submental incision. Now the connector on the endotracheal tube was removed and the tube was exteriorized via the submental tract and connected to the ventilator (Figures 3-8). The tube was secured to the skin with 1.0 silk suture. Anesthesia was maintained using nitrous oxide and oxygen at a ratio of 60:40 and intermittent doses of intravenous vecuronium to provide muscle relaxation.

At the end of the surgical procedure, the tube connector was removed. The pilot tube and the endotracheal tube are passed back through the incision in to the mouth, reversing the original path. The submental wound was sutured using 3.0 silk sutures. Reversal of anaesthesia was achieved using glycopyrollate and neostigmine intravenously. The parameters like time taken for the procedure, surgical access ,associated hemorrhage, accidental partial extubation of endotracheal tube, complications like salivary fistula, damage to the marginal mandibular branch of facial nerve, damage to lingual nerve, wound infection and post operative scarring were noted.

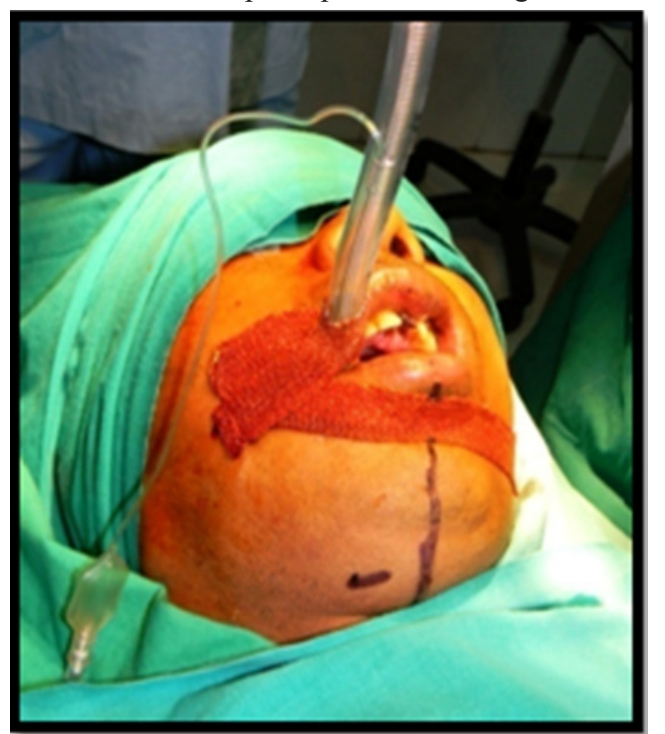

Figure I Oral intubation with submental marking

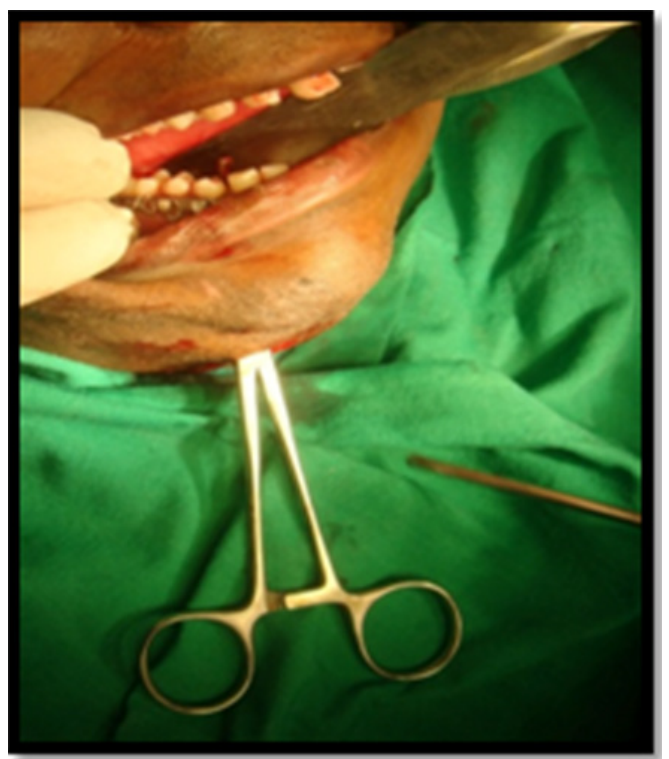

Figure 2 Blunt dissection.

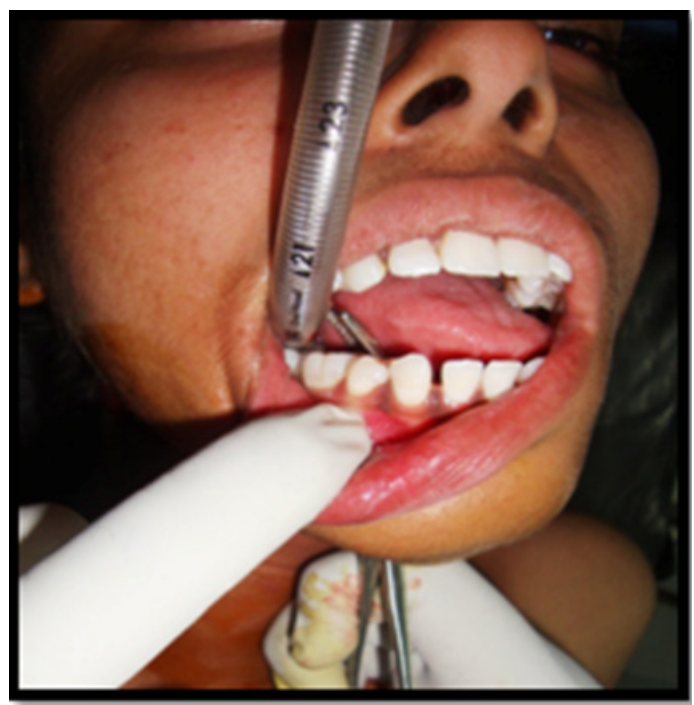

Figure 3 Oral to submental shift of tube.

\section{Results}

Patient's clinical data are presented in Tables $1 \& 2$. In all subjects, submental intubation allowed simultaneous treatment of all fractures and elective orthognathic procedures without changing the method of intubation and without any interference from the tube during the operation. There was no difficulty in passing the tube through the floor of the mouth, and the total duration of the submental procedure ranged from 5-12 minutes (mean of 7 minutes).

Disconnection of the standard connector from the tube was done easily. There was no significant oxygen desaturation in any subject during the procedure. The intubation method itself did not lead to any intraopertaive complications except in case 5 of trauma group where pilot balloon puncture was noticed and was managed by clamping with hemostat. Intraoperative assessment of nasolabial complex was found easy in elective orthognathic procedures and widening of Alar base after AMO and Lefort 1 osteotomy were minimized. Significant 
hemorrhage was not encountered using the paramedian approach in any of the subjects. Wound dehiscence was observed in 2 cases of trauma due to superficial infection, sutures breakdown which resolved after using topical application of antibiotics and delayed suturing. None of the patients in the study required postoperative ventilation. All the patients were evaluated 1 week, 1 month and 6 months. No motor or sensory salivation deficit was found. Post operative scar is almost imperceptible and less conspicuous.

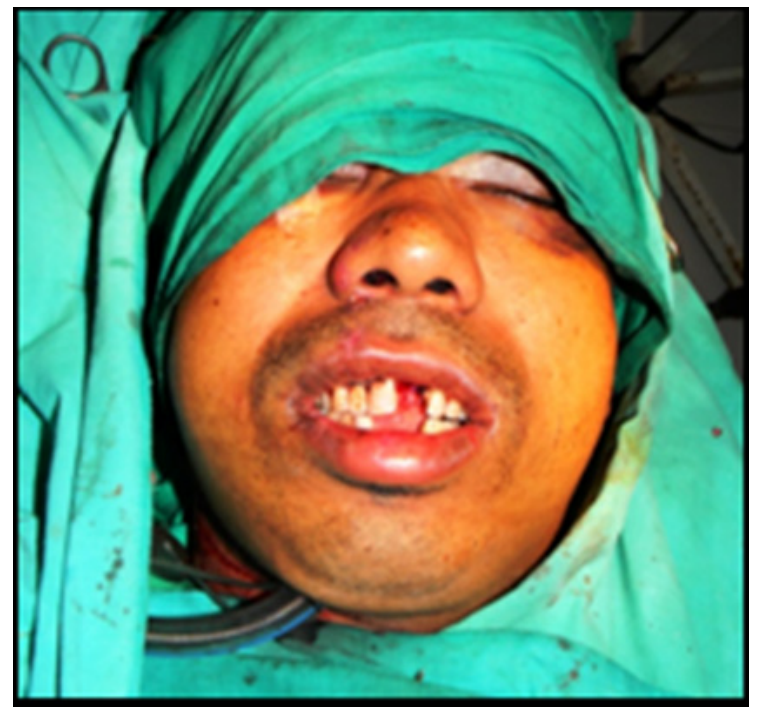

Figure 4 Trauma - Lateral Submental Intubation.

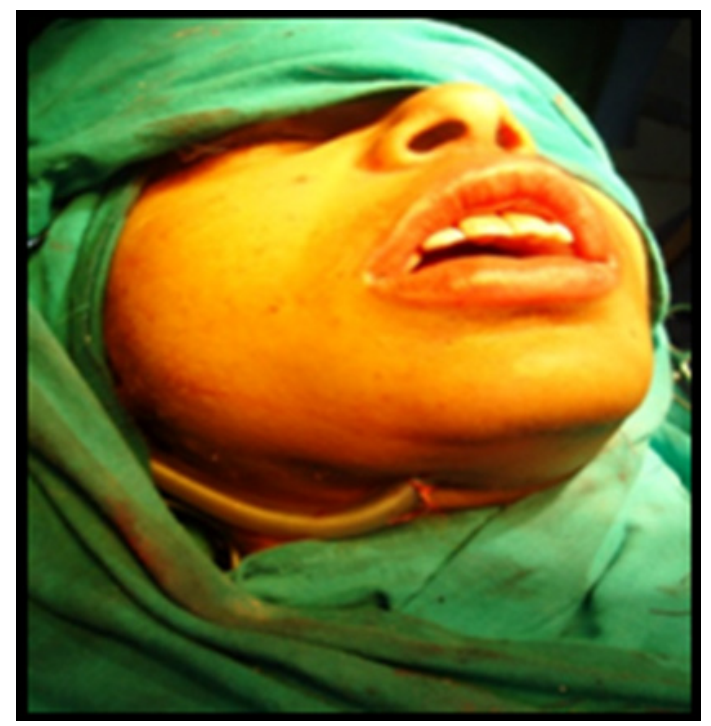

Figure 5 Orthognathic Lateral Submental Intubation.

\section{Discussion}

Injuries to the Head and Maxillofacial areas can easily jeopardize the patient's ability to maintain the airway. Management of the airway is always a primary concern during any maxillofacial surgical procedures. Operating in the field free from intubation is comfortable for a surgeon; while for an anesthesiologist, the safety of the endotracheal tube and efficiency of ventilation are important. Endotracheal intubation by means of submental route was first described by Altemir in $1986 .^{7}$ This technique provides a secure airway, an unobstructed intra oral surgical field and allows maxillo- mandibular fixation avoiding the drawbacks and complications of oral and nasal intubation as well as tracheostomy. Despite the widespread use of submental intubation for other purposes, it was Bogi and Incze in 1996 who recommended the use of submental intubation in elective osteotomies. They advocated paramedian approach so that the geniohyoid and the genioglossus muscles do not have to be crossed and easily sparing the insertion of the anterior belly of digastric muscle. ${ }^{10}$

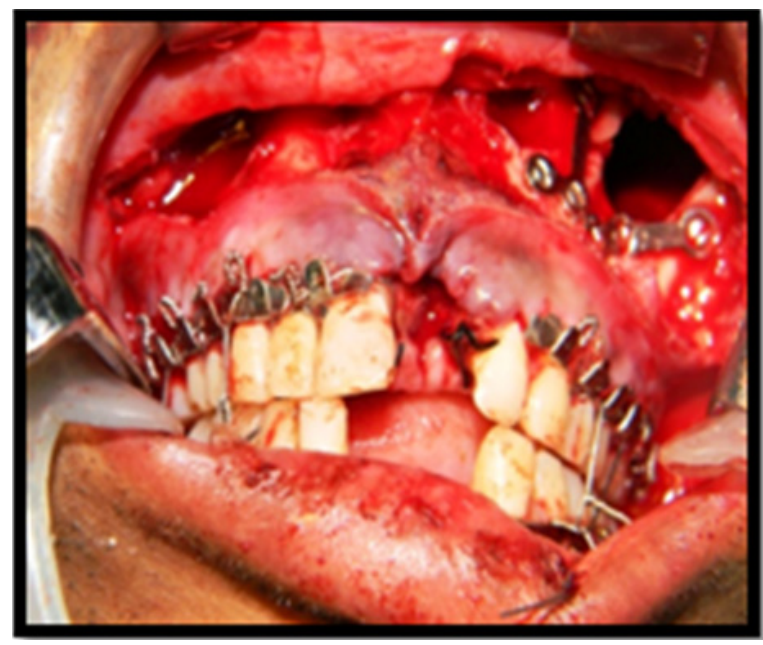

Figure 6 Intraoperative IMF and plate fixation.

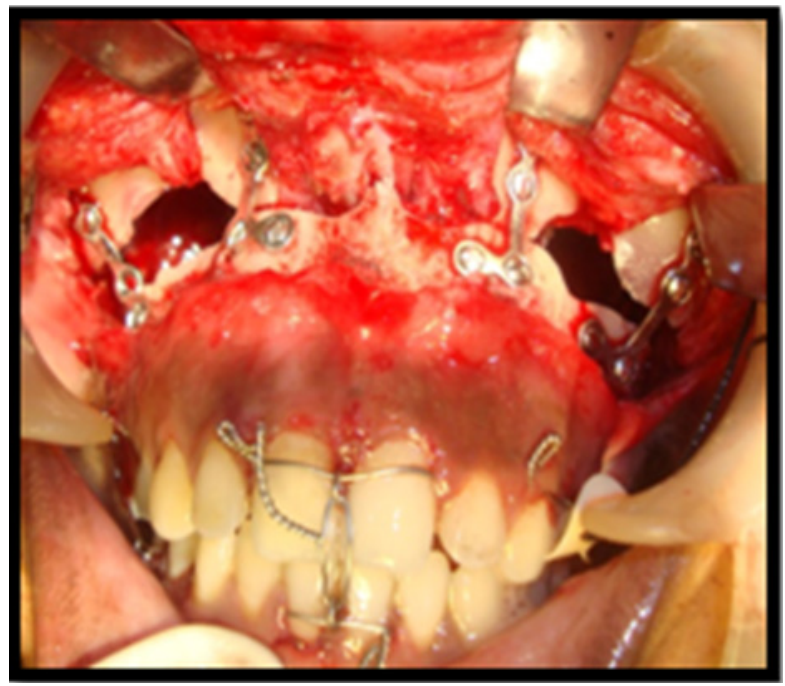

Figure 7 Lefort I Osteotomy with IMF.

In 30 patients who require correction of dentofacial deformities, conventional endonasal intubation was avoided as it would be difficult to assess precise intra-operative changes to the nasolabial complex, assessment of midlines, cant and incisor display accurately during surgical procedures without changing the tube. Oral intubation in all these patients was not preferred as the endotracheal tube interferes with the intraoperative maneuvers of restoring the occlusion by means of intraoperative maxillomandibular fixation. Of 60 patients, Paramedian approach described by Altemir where dissection was done subperiosteally was not followed in our study. The paramedian approach described by Altemir involving subperiosteal dissection was avoided in our study. Instead, a paramedian approach with extraperiosteal blunt dissection keeping as close as possible to the inner side of the mandible as advocated by Biglioli $\mathrm{F}^{11}$ and Taglialatela 
Scafati was adopted in all 60 cases. ${ }^{12,13}$ The endotracheal tube passes close to the anterior belly of digastric muscle, anterior most portion of the mylohyoid muscle and the lower edge of the mandible.

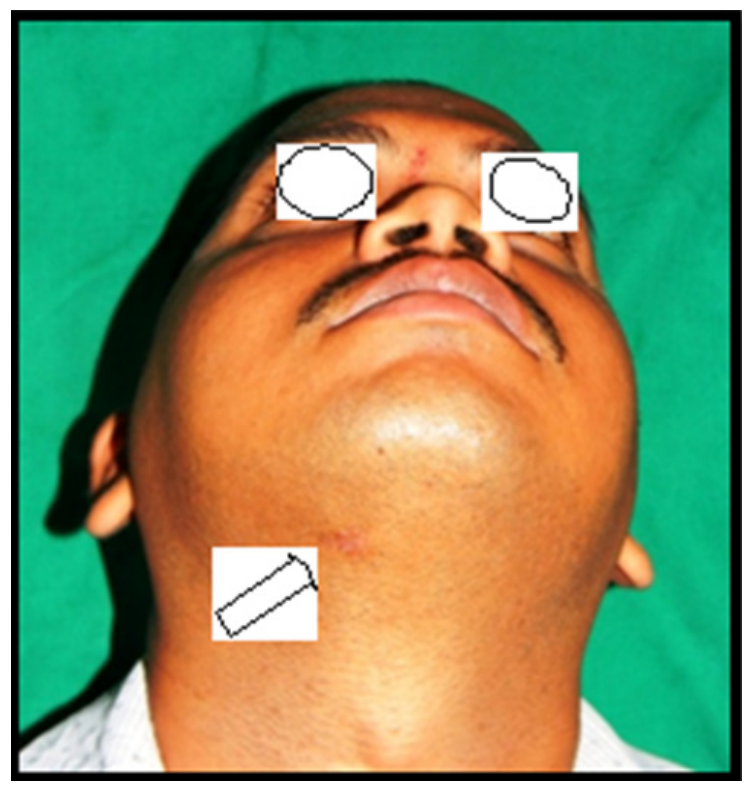

Figure 8 Trauma -post operative scar.

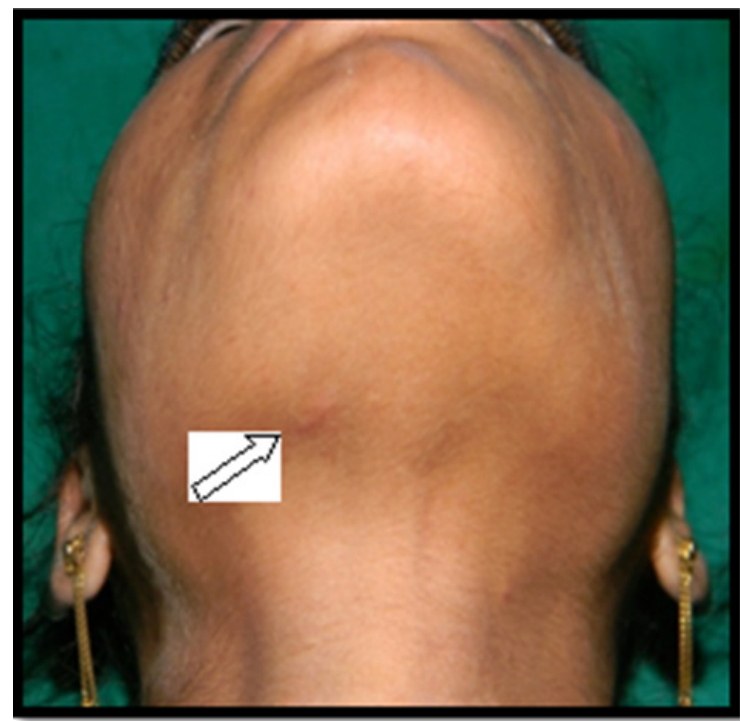

Figure 9 Trauma -post operative scar.

Some factors must be considered to make submental endotracheal intubation a successful technique with minimal morbidity. Good communication between surgeon and anesthetist is mandatory at every step. Initial management of the airway of patient with facial trauma can be challenging. The submental intubation is always a second step and precaution must be taken to firmly secure the endotracheal tube intraorally to prevent accidental extubation. To avoid injury to the glands and ducts, blunt dissection with hemostat must run in close approximation to the medial border of the mandible and once intubated the tube should be secured with sutures. The time taken for the procedure was an average of 7 minutes when compared to other procedures described in the literature.

The morbidity associated with this technique is very low, with no episodes of compromised airway or arterial desaturation occuring during the procedure. 5 cases of superficial wound infection occurred that fully resolved after a course of appropriate antibiotics. Other potential complications like oro-cutaneous fistula, trauma to the sublingual and submandibular glands or ducts, damage to the lingual nerve, hypertrophic scar or mucocele as mentioned in literature were not encountered in our study. ${ }^{14}$

Submental route is a quick, simple, safe and acceptable alternative to tracheostomy for short term airway management in patients with facial trauma and elective orthognathic surgeries with clear defined advantages. The present study reports good results with use of submental endotracheal intubation for surgical treatment of 60 patients with maxillofacial trauma and orthognathic surgery without interference from the artificial airway and most importantly without compromising the airway.

\section{Conclusion}

Our series of 60 cases reestablishes that submental intubation is a useful alternative technique of airway management not only in patients with maxillofacial trauma but also in elective orthognathic surgery. It demands a certain surgical skill without specialized equipments; however, it is safe and quick to execute. It allows intraoperative correction of occlusion and enables surgery for dentofacial deformities and avoids the dangers of classical endotracheal intubation.

The main advantages of this technique are that

a. The technique is simple and requires no other special equipment than the normally used instruments.

b. The endotracheal tube does not interefere with intraoperative maneuvers like fracture reduction and stabilization and ensures an unobstructed intraoral surgical field.

c. The possibility of contaminating the operative field is minimal.

d. The flow of anaesthestic gases are evenly maintained during the course of anaesthesia

e. Leaves a negligible scar in the submental region.

f. This technique, when used in appropriate cases, allowed both the surgeon and the anaestheist to deliver a better quality of patient care.

\section{Acknowledgments}

None.

\section{Conflicts of interest}

The authors declare there is no conflict of interests.

\section{Funding}

None.

\section{References}

1. Hall C E J, Shutt LE. Nasotracheal intubation for head and neck surgery. Anaesthesia. 2003;58:249-256.

2. Coe TR, Human M. The peri-operative complications of nasal intubation. Anaesthesia. 2001;56(5):447-484.

3. Gibbons AJ, Hope DA, Silvester KC. Oral endotracheal intubation in the management of midfacial fractures. Br J Oral Maxillofac Surg. 2003;41(4):259-260.

4. Suda N, Murakami C, Kawamoto T, et al. Three cases of anterior maxillary osteotomy under orotracheal intubation. Int $J$ Adult orthod Orthognath Surg. 2002;17(4):273-282. 
5. Castling B, Telfer M, Avery BS. Complications of tracheostomy in major head and neck cancer surgery: A retrospective study of 60 consecutive cases. Br J Oral Maxillofac Surg. 1994;32(1):3-5.

6. Halfpenny W, McGurk M. Analysis of Tracheostomy-Associated Morbidity after Operations for Head and neck Cancer. $\mathrm{Br} \mathrm{J}$ Oral Maxillofac Surg. 2000;38(5):509-512.

7. Francisco He.rnandez Altemir. The submental approach for tracheal intubation: a new technique. Journal of Maxillofacial Surgery. 1986;14:64-65

8. Gordon NC, Tolstunov L. Submental approach to oro endotracheal intubation in patients with midfacial fractures. Oral Surg Oral Med Oral Path Oral Radiol Endod. 1995;79(3):269-272.

9. Chandu A, Witherow H, Stewart A. Submental intubation in orthognathic surgery: initial experience. Br J Oral Maxillofac Surg. 2008;46(7):561563.
10. Nyárády Z, Sári F, Olasz L, et al. Ubmental endotracheal intubation in concurrent orthognathic surgery: A technical note. J Craniomaxillofac Surg. 2006;34(6):362-365.

11. Biglioli F, Mortini P, Goisis M, et al. Submental orotracheal intubation:an alternative to tracheotomy in transfacial cranial base surgery. Skull Base. 2003;13(4):189-195.

12. Taglialatela Scafati C, Maio G, Aliberti F, et al. Submento-submandibular intubation: is the subperiosteal passage essential? experience in 107 consecutive cases. Br J Oral Maxillofac Surg. 2006;44(1):12-14.

13. Taglialatela Scafati C. Mucoceles as a complication of submandibular intubation. J Cranio Maxillofac Surg. 2004;32:335.

14. Stranc MF, Skoracki R. A Complication of submandibular intubation in a panfacial fracture patient. J Maxillofac Surg. 2001;29(3):174-176. 